\title{
LTR-mediated retroposition as a mechanism of RNA-based duplication in metazoans
}

\author{
Shengjun Tan, ${ }^{1}$ Margarida Cardoso-Moreira, ${ }^{2,6}$ Wenwen Shi, ${ }^{1}$ Dan Zhang, ${ }^{1,3}$ \\ Jiawei Huang, ${ }^{1,3}$ Yanan Mao, ${ }^{1}$ Hangxing Jia, ${ }^{1,3}$ Yaqiong Zhang, ${ }^{1}$ Chunyan Chen, ${ }^{1,3}$ \\ Yi Shao, ${ }^{1,3}$ Liang Leng, ${ }^{1}$ Zhonghua Liu, ${ }^{4}$ Xun Huang, ${ }^{4}$ Manyuan Long, ${ }^{5}$ \\ and Yong E. Zhang ${ }^{1,3}$ \\ ${ }^{1}$ Key Laboratory of Zoological Systematics and Evolution and State Key Laboratory of Integrated Management of Pest Insects and \\ Rodents, Institute of Zoology, Chinese Academy of Sciences, Beijing 100101, China; ${ }^{2}$ Center for Integrative Genomics, University of \\ Lausanne, 1015 Lausanne, Switzerland; ${ }^{3}$ University of Chinese Academy of Sciences, Beijing 100049, China; ${ }^{4}$ State Key Laboratory \\ of Molecular Developmental Biology, Institute of Genetics and Developmental Biology, Chinese Academy of Sciences, Beijing 100101, \\ China; ${ }^{5}$ Department of Ecology and Evolution, The University of Chicago, Chicago, Illinois 60637, USA
}

\begin{abstract}
In a broad range of taxa, genes can duplicate through an RNA intermediate in a process mediated by retrotransposons (retroposition). In mammals, L1 retrotransposons drive retroposition, but the elements responsible for retroposition in other animals have yet to be identified. Here, we examined young retrocopies from various animals that still retain the sequence features indicative of the underlying retroposition mechanism. In Drosophila melanogaster, we identified and de novo assembled 15 polymorphic retrocopies and found that all retroposed loci are chimeras of internal retrocopies flanked by discontinuous LTR retrotransposons. At the fusion points between the mRNAs and the LTR retrotransposons, we identified shared short similar sequences that suggest the involvement of microsimilarity-dependent template switches. By expanding our approach to mosquito, zebrafish, chicken, and mammals, we identified in all these species recently originated retrocopies with a similar chimeric structure and shared microsimilarities at the fusion points. We also identified several retrocopies that combine the sequences of two or more parental genes, demonstrating LTR-retroposition as a novel mechanism of exon shuffling. Finally, we found that LTR-mediated retrocopies are immediately cotranscribed with their flanking LTR retrotransposons. Transcriptional profiling coupled with sequence analyses revealed that the sense-strand transcription of the retrocopies often lead to the origination of in-frame proteins relative to the parental genes. Overall, our data show that LTR-mediated retroposition is highly conserved across a wide range of animal taxa; combined with previous work from plants and yeast, it represents an ancient and ongoing mechanism continuously shaping gene content evolution in eukaryotes.
\end{abstract}

[Supplemental material is available for this article.]

Retrotransposons are pervasive in eukaryotic genomes and consist of two major classes: long terminal repeats (LTRs) and non-LTR retrotransposons (mainly long interspersed nuclear elements or LINEs). In each class, they can be further divided into autonomous and nonautonomous retrotransposons. Autonomous retrotransposons encode the proteins required for their own mobilization, whereas nonautonomous retrotransposons are trans-mobilized by the autonomous elements. For example, in mouse nonautonomous MaLR, elements can be copied by the autonomous MERVL elements since they share similar LTRs (McCarthy and McDonald 2004). A key contribution of retrotransposons to evolution is their capacity to mediate the formation of new genes in a process termed retroposition (Soares et al. 1985; Brosius 1991; Eickbush 1999). Herein, the retrotransposon machinery reverse transcribes messenger RNAs (mRNAs) into complementary DNAs (cDNAs) and inserts them back into the genome as retrocopies

\footnotetext{
6Present address: Zentrum für Molekulare Biologie der Universität Heidelberg (ZMBH), 69120 Heidelberg, Germany

Corresponding author: zhangyong@ioz.ac.cn

Article published online before print. Article, supplemental material, and publication date are at http://www.genome.org/cgi/doi/10.1101/gr.204925.116. Freely available online through the Genome Research Open Access option.
}

(Kaessmann et al. 2009). Unlike DNA-based duplicates, functional retrocopies or retrogenes inherently lack the ancestral regulatory sequences, having to recruit new ones from the vicinity and/or by evolving them de novo (Kaessmann et al. 2009; Carelli et al. 2016). The contribution of retrogenes to phenotypic evolution is well recognized ranging from the avoidance of male-male courtship in fruit fly to the short-legged phenotype in domestic dogs (Parker et al. 2009; Chen et al. 2013).

Currently, our understanding of the mutational mechanisms underlying retroposition is largely limited to mammalian systems in which it has been conclusively shown that retrocopies can be generated by the reverse transcriptase (RT) of LINE1 or L1 retrotransposons (Eickbush 1999; Moran et al. 1999; Esnault et al. 2000; Wei et al. 2001). L1-mediated retrocopies harbor several hallmark sequences in their flanking regions: a TTTT/AA endonuclease cleavage site, a poly(A) tail priming the reverse transcription, target site duplications (TSDs) generated when fixing the insertion nick overhang, and a bias favoring truncation of the $5^{\prime}$ rather than the 3' end (Kaessmann et al. 2009; Richardson et al. 2014).

C 2016 Tan et al. This article, published in Genome Research, is available under a Creative Commons License (Attribution-NonCommercial 4.0 International), as described at http://creativecommons.org/licenses/by-nc/4.0/. 
In principle, LTR retrotransposons could also mediate the formation of retrocopies. Specifically, LTR retrotransposons have a close evolutionary relationship with retroviruses (Wicker et al. 2007), which replicate by undergoing two rounds of template switches during their viral DNA synthesis and use a low affinity RT enzyme (Delviks-Frankenberry et al. 2011). Retroviruses can capture host mRNAs (Hajjar and Linial 1993), and their RT can use the mRNA as a template through switching induced by microsimilarity (between short similar sequences shared by the mRNA and the retrovirus, also called microhomology) (Goodrich and Duesberg 1990; Luo and Taylor 1990). Sometimes sequence similarity-independent mechanisms (e.g., 3' transduction) (Swain and Coffin 1992) may also work. Thus, it is conceivable that mRNAs could be captured in the virus-like particles (VLPs) where LTR retrotransposons replicate (Yoshioka et al. 1990; Havecker et al. 2004), and the latter could mediate the retroposition of these mRNAs in a manner analogous to that of retroviruses. The evidence for this process is, however, very limited. In yeast, reporter assays demonstrated that the LTR retrotransposon Ty can capture partial mRNA sequences and form a chimera consisting of internal retrocopies flanked by the retrotransposon, and this is often accompanied by microsimilarity at the fusion points (Derr et al. 1991; Schacherer et al. 2004). In maize, a chimera consisting of partial sequences derived from three unlinked cellular genes flanked by the LTR retrotransposon Bs1 has been described (Elrouby and Bureau 2001, 2010); in rice, 27 retrocopies were found to be located within LTR retrotransposons (Wang et al. 2006). Finally, a single case has been described in human in which the LTR retrotransposon HERV-K captured the gene FAM8A1 (with microsimilarity present at the fusion points), resulting in a currently untranscribed processed pseudogene Jamain et al. 2001).

Here, we performed a genome-wide study across a broad range of taxa (fruit fly, mosquito, zebrafish, chicken, mouse, and human) to determine whether LTR retrotransposons can generally drive retroposition in animals and to identify the features associated with LTR-mediated retrocopies.

\section{Results}

De novo assembly reveals a conservative but highly reliable data set of 15 polymorphic retrocopies in the Drosophila melanogaster Genetic Reference Panel (DGRP)

We started our analyses by focusing on polymorphic retrocopies, which are encoded by at least one inbred line but are absent from the reference genome. Specifically, we analyzed the 40 core DGRP lines whose average sequencing depth reaches $52 \times$ (Supplemental Table 1). Based on previous work (Schrider et al. 2011, 2013; Ewing et al. 2013), we developed a refined pipeline to identify polymorphic retrocopies that combines both exonexon and exon-intron junction read mapping and that includes targeted local de novo assembly (Methods; Supplemental Fig. 1).

We identified a total of 31 candidate retrocopies supported by more than one read. Of these, we assembled 15 retrocopies and their flanking regions (Table 1; Supplemental Table 2; Supplemental Data Set 1). The remaining 16 candidate retrocopies could not be assembled because of the low sequencing depth of the retroposed loci (for 12 candidate retrocopies) (Supplemental Table 3) or because of mapping problems (for four retrocopies). We chose to focus on the set of 15 retrocopies because knowing their flanking regions is critical to infer the underlying mutational mecha-
Table 1. The 15 D. melanogaster polymorphic retrocopies

\begin{tabular}{|c|c|c|c|}
\hline $\begin{array}{l}\text { Parental } \\
\text { gene }\end{array}$ & $\begin{array}{l}\text { Parent/ } \\
\text { retrocopy } \\
\text { transcript } \\
\text { length (bp) }\end{array}$ & $\begin{array}{c}\text { LTR } \\
\text { retrotransposon } \\
\text { annotation }\end{array}$ & $\begin{array}{l}\text { Microsimilarity } \\
\text { (bp) }\end{array}$ \\
\hline CG17604 & $2651 / 975$ & BLASTOPIA, Gypsy & 16,2 \\
\hline CG10212 & $3687 / 1323$ & BLASTOPIA, Gypsy & 6,0 \\
\hline CG32082 & $5246 / 704$ & BLASTOPIA, Gypsy & 13,1 \\
\hline $\begin{array}{l}\text { CG4799, } \\
\text { CG11924 }\end{array}$ & $\begin{array}{r}2571 / 243 \\
3530 / 917\end{array}$ & BATUMI, Pao & $6,9,12$ \\
\hline $\begin{array}{l}\text { CR42443, } \\
\quad \text { CG5119 }\end{array}$ & $\begin{array}{l}1023 / 441, a \\
3127 / 1294\end{array}$ & STALKER2, Gypsy & $2,-16$ \\
\hline CG33957 & $3879 / 745$ & BLASTOPIA, Gypsy & 9,1 \\
\hline CG14796 & $5388 / 1073$ & MAX, Pao & 10,10 \\
\hline CG31605 & $2792 / 1564^{\mathrm{a}}$ & TABOR, Gypsy & 9 \\
\hline CG8567 & $2425 / 1683$ & 412, Gypsy & $-2,3$ \\
\hline CG2662 & $1647 / 377$ & 412, Gypsy & $-26,15$ \\
\hline CG5020 & $5625 / 1469$ & BLASTOPIA, Gypsy & 7,2 \\
\hline CG3631 & $2757 / 1695$ & Gypsy5, Gypsy & 4,7 \\
\hline CG1242 & $2713 / 915$ & MAX, Pao & 16,2 \\
\hline CG10652 & $513 / 205$ & 412, Gypsy & 16,1 \\
\hline CG8392 & $846 / 394^{\mathrm{a}}$ & 412, Gypsy & 12 \\
\hline
\end{tabular}

Transcript length is the length of the parental gene's mRNA. When a parental gene encodes multiple alternative isoforms compatible with the retroposed sequences, one isoform is randomly chosen (see Supplemental Data Set 1). The LTR retrotransposon is described as the element name followed by its family name, e.g., BLASTOPIA, Gypsy. Microsimilarity refers to the stretch of similar sequence present at the switch points shared by the retrocopies and the flanking LTR retrotransposons. The numbers in this column follow the order of retroposition, i.e., from retrotransposons' $3^{\prime}$ terminal to their $5^{\prime}$ terminal. The three negative values refer to the length of de novo sequences inserted at the switch point. For the retrocopy derived from CG10212, there is no microsimilarity or de novo sequence at its $5^{\prime}$ end, therefore, it is referred to as " 0. "

${ }^{\mathrm{a}}$ Retrocopies for which we failed to assemble one flanking region. The length of the current contig is shown.

nism. For 10 of the 15 retrocopies for which we could design probes, we confirmed their existence by PCR experiments (Methods; Supplemental Fig. 2; Supplemental Table 4). We further randomly chose three retrocopies for Sanger sequencing and confirmed the assembled sequences (Supplemental Data Set 2).

Based on the presence/absence of exon-exon junction reads across the 40 lines, we estimate the lower-bound population frequencies of the 15 retrocopies to range from 1 to 31 (Supplemental Table 3). Notably, for both CG4799_CG11924_r and CG5119_CR42443_r, the sequences of two parental genes were retroposed together generating new chimeric gene structures, each containing the partial sequences of two unlinked genes. In total, 17 parental genes were involved in the formation of 15 polymorphic retrocopies (Table 1).

\section{LTR retrotransposons mediate retroposition in Drosophila populations}

The 15 retrocopies tend to be partial with a median length of $26 \%$ of their parental transcripts' size, a consequence of both $5^{\prime}$ and $3^{\prime}$ truncations with the latter being more extensive (median truncation $419 \mathrm{bp}$ vs $1387 \mathrm{bp}$, Wilcoxon rank test $P=0.04$ ) (Supplemental Table 2). In addition to the lack of the 5' truncation bias, all other hallmarks suggestive of L1-mediated retroposition (i.e., presence of a poly[A] tail, TSDs, and a recognition site) are absent from the 15 retrocopies. When we aligned the retrocopies' flanking sequences against the reference genome, we found that 
all flanking sequences consisted of LTR retrotransposons (Table 1). Most notably, this association was not created by the insertion of the retrocopies into preexisting LTR retrotransposons but instead by recombination events mediated by microsimilarity between the retrocopies and the LTR retrotransposons. Specifically, no retrotransposons flanking the retrocopies showed the signature of a previously continuous element that was interrupted by the insertion of a foreign sequence. Instead, in all cases, a portion of the internal retrotransposon open reading frames (ORFs) was replaced by the retrocopies (Supplemental Data Set 1). Moreover, for 25 of the 29 (86\%) junctions identified, we found stretches of microsimilarity shared between the LTR retrotransposons and the mRNAs (median length of 6 bp) (Table 1; Supplemental Data Set 1). For three junctions, de novo sequences (2-26 bp) were found inserted between the two elements. Simulations show that the extent of microsimilarity cannot be explained by the random insertion of retrocopies within LTR retrotransposons (Methods; Supplemental Fig. 3).

Thus, retroposition in Drosophila seems to occur via a microsimilarity-dependent template switch mechanism of LTR retrotransposons, which is analogous to the one reported for retroviruses and yeast (Goodrich and Duesberg 1990; Luo and Taylor 1990; Derr et al. 1991; Hajjar and Linial 1993; Schacherer et al. 2004). One implication of this mechanism is that both $5^{\prime}$ and $3^{\prime}$ LTRs should coexist in the retroposed product because the integrase has to recognize 15 - to 20-bp sequences located at each terminal (Hindmarsh and Leis 1999; Engelman et al. 2009). Consistently, we found in the assembled sequences of nine (60\%) retrocopies, the presence of the 5' LTR (the data are unclear for the other retrocopies because the assembled sequences are too short). In addition, for five retrocopies, for which we obtained sequences at the two terminals, we confirmed the presence of both $5^{\prime}$ and 3' LTRs (Methods; Supplemental Data Sets 3-5). Although var- ious families of LTR retrotransposons are associated with the retrocopies (Table 1), for the five retrocopies associated with the Gypsy BLASTOPIA, we found a unique pattern: The RT always switches back from the mRNA to the retrotransposon at one particular site, TAAAAAACAAGC-GGTTG, which is always near an exonexon junction (Fig. 1A; Supplemental Data Set 1). Our data also show that more than two switches can occur. For example, a segment from the 5' untranslated region (UTR) of CG4799 and a segment from the coding sequence (CDS) of CG11924 were coretroposed by the Pao BATUMI element to form a new chimeric retrocopy, in which all three switch points are associated with stretches of microsimilarity (Fig. 1B). Thus, two or more genes can be involved in this process to form a chimeric gene, which could be viewed as a new mechanism of exon shuffling (Gilbert 1978; Long et al. 1995; Li 1997).

In Drosophila, $44 \%$ of recently inserted LTR retrotransposons are located within or nearby genes, which is an overrepresentation compared to a random insertion model (Ganko et al. 2006). One would expect that retrocopies created by LTR retrotransposons should show a similar pattern. We could not test this hypothesis with the assembled retrocopies, because they do not include the sequences outside the flanking LTR retrotransposons. So, we generated whole-genome sequencing data with longer read sizes and mobile element targeted sequencing data via the ME-Scan methodology (Methods; Supplemental Table 5; Witherspoon et al. 2010). With these additional data, we identified 18 insertion sites for nine retrocopies (Table 2; Supplemental Table 6). Twelve insertion sites were further validated by PCR followed by Sanger sequencing. In agreement with the biased insertion pattern of LTR retrotransposons, most (8/12) retrocopies were inserted within genes.

Intriguingly, two retrocopies (CG17604_r and CG10212_r) are associated with more than one insertion site (Table 2),

A

CDS of CG17604

GCACAAAAGGAAAAGTTTGAGGCAAACATTTTACAATTAAGCA......GAGCATTCCTACTAAGGTGCAACCGGAAAATACAGACGAATTTTCAAGCACTGCCAG GAAGCAGATAAAAAACAAGCGG TTGAGTATTCCCAAATGTTT ATGAAGTTGTGGAAGTACATAGCCG AAAATGCAGAC CTATTGTCATCCGGGTCGGA 5' BLASTOPIA

3' BLASTOPIA

B

3' BATUMI

TCTCCGTGTTGATGCCTGACAGTTCTAAGGCAGTCAAGCAACCTTG

GCTTTTCAAATCAAACGGTAAATTCTGGCGGCGCCAAGCAGGCATT....... - ר

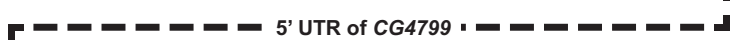

- - -....AAAGTTTCACACATTTCATCGCAGCAGCAAACATCTAAACTTTTAAAGA

CAACAACAACAGCAGCAGCAGCAGCAGCAGGAACTGCTGGAGCAGCAGC.....- - ר

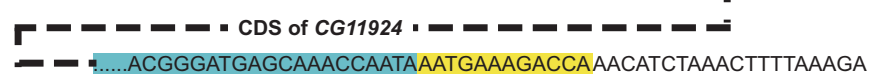

GGAAAATTCCTAGTTTGCTGAAAAAAAGACCACTCGAAAAAACACTCGAAGA

5' BATUMI

C

CDS of AGAP000264

AACGCTGCTCGAACGCAACAAAGACCTGGAGGCGACG......AGCTCAAGAGCACGCAAAAGTGTCTCCTGTCCGAG

TCCTGTCTTTAATCCCAACAAACCAGGAAAGGTGCGA TCGTACAGTCGGTAAAAAAGGTGTTAAGAGAGATA

5' BEL18-I_AG

3' BEL18-I_AG

D

CDS of ENSMUSG00000021752

TCGCGCTCTCGGGCCGCCGG CGTCTCCTGGCCAGGTCG.......GTCCACTCCTTACTCGAAGGCATCTCAAACTATTTCACCAAGTGGAAT CCCACTTTATAGTGACTGCCAT TTATGGCCGGCCTTGA AAGACCTCTTTTACTGGCATCTCAAGGTTACTGGAACTGCTCCAA

5' IAPEY

3' IAPEY

Figure 1. Schematic representations of a subset of the retrocopies identified. (A) CG17604_r in D. melanogaster. (B) CG4799_CG11924_r in D. melanogaster. (C) ENSANGG00000011308 in mosquito. (D) ENSMUSG00000083549 in mouse. LTR retrotransposons and retrocopies are marked in gray and blue, respectively. Microsimilarity stretches are marked in yellow, and de novo sequence insertions in green. The red line in $A$ marks the "TAAAAAACAAGC-GGTTG" motif. (CDS) coding sequences; (UTR) untranslated regions. 
Table 2. Insertion sites of nine retrocopies

\begin{tabular}{|c|c|c|c|}
\hline \multirow{2}{*}{$\frac{\text { Retrocopy }}{\text { CG17604_r }}$} & \multicolumn{2}{|c|}{ Position } & \multirow{2}{*}{$\frac{\text { Method }}{2 \times 250 \mathrm{bp}}$} \\
\hline & SR:TATAA & & \\
\hline & Satellite $^{\mathrm{a}}$ & & $2 \times 250 \mathrm{bp}$ \\
\hline & 2L:11489564 ${ }^{\mathrm{a}}$ & Intergenic & $\begin{array}{l}2 \times 250 \text { bp } \\
\text { and ME- } \\
\text { Scan }\end{array}$ \\
\hline & $2 R: 21009193^{a}$ & $\begin{array}{l}\text { Intronic of } \\
\text { CG16778 }\end{array}$ & ME-Scan \\
\hline & 3R: $3618946^{\mathrm{a}}$ & $\begin{array}{l}3^{\prime} \text { UTR of } \\
\text { CG10086 }\end{array}$ & ME-Scan \\
\hline & $3 R: 9536417^{a}$ & $\begin{array}{l}\text { Intronic of } \\
\text { CG33748 }\end{array}$ & ME-Scan \\
\hline CG10212_r & LINE:DOC_DM & & $2 \times 250 \mathrm{bp}$ \\
\hline & 2L:13226230 & Intergenic & ME-Scan \\
\hline & $2 \mathrm{R}: 3607631^{\mathrm{a}}$ & $\begin{array}{l}\text { Intronic of } \\
\quad \text { CG18812 }\end{array}$ & $2 \times 250 \mathrm{bp}$ \\
\hline & $3 R: 21723045^{a}$ & Intergenic & $2 \times 250 \mathrm{bp}$ \\
\hline CG32082_r & $3 \mathrm{~L}: 230088887^{\mathrm{a}}$ & $\begin{array}{r}\text { Intronic of } \\
\text { CG8779 }\end{array}$ & ME-Scan \\
\hline CG4799_CG11924_r & $3 L: 21967546^{a}$ & $\begin{array}{r}\text { Intronic of } \\
\text { CG7470 }\end{array}$ & $\begin{array}{l}2 \times 250 \text { bp } \\
\text { and ME- } \\
\text { Scan }\end{array}$ \\
\hline CG33957_r & $2 \mathrm{~L}: 17134768^{\mathrm{a}}$ & $\begin{array}{l}\text { Intronic of } \\
\quad \text { CG34106 }\end{array}$ & ME-Scan \\
\hline CG14796_r & $\begin{array}{r}X: 8740722- \\
8741237^{b}\end{array}$ & $\begin{array}{l}\text { Intronic of } \\
\quad \text { CG12075 }\end{array}$ & ME-Scan \\
\hline CG31605_r & $3 \mathrm{~L}: 17668235^{\mathrm{b}}$ & $\begin{array}{l}\text { Intronic of } \\
\text { CG34251 }\end{array}$ & ME-Scan \\
\hline CG5020_r & $3 R: 13789450^{a}$ & $\begin{array}{l}\text { Intronic of } \\
\text { CG42457 }\end{array}$ & $\begin{array}{l}\text { mate pair } \\
\text { and ME- } \\
\text { Scan }\end{array}$ \\
\hline CG1242_r & $\begin{array}{c}3 L: 2624322- \\
2624753^{\mathrm{b}}\end{array}$ & $\begin{array}{l}\text { Intronic of } \\
\quad \text { CG12002 }\end{array}$ & \\
\hline & $\begin{array}{c}3 R: 15303121- \\
15303991^{\mathrm{b}}\end{array}$ & $\begin{array}{l}\text { Exonic/ } \\
\text { intronic of } \\
\text { CG34157 }\end{array}$ & ME-Scan \\
\hline
\end{tabular}

All insertion sites except CG17604_r in a simple repeat (SR) and CG10212_r in a LINE were tested by PCR and Sanger sequencing.

Validated sites.

${ }^{\mathrm{b}}$ False positives. although they appear to be derived from a single template switch event because they share the same gene structure. For CG17604_r, the evidence for a single template switch event is even stronger, because the different retrocopies share eight nucleotide differences that distinguish them from the parental gene (Supplemental Data Set 6 ). Considering that $>80 \%$ of DNA-based segmental duplication (SD) occurs in tandem in D. melanogaster (Zhou et al. 2008), the presence of multiple identical retrocopies located dispersedly in the genome is unlikely to be a consequence of independent SDs. One possible scenario is that the whole locus acts as a nonautonomous LTR retrotransposon that can be further retroduplicated by the enzymes of autonomous LTR retrotransposons. This scenario is not unlikely considering that nonautonomous LTR retrotransposons are much more likely to proliferate than their corresponding autonomous elements, presumably because the former are less likely to be recognized as retroviruses and thus repressed by the host machinery (Maksakova et al. 2006).

In summary, we have described three lines of evidence that identify LTR retrotransposons as capable of mediating retroposition in Drosophila: (1) All polymorphic retrocopies are flanked by LTR retrotransposons, and the switch points between the LTR retrotransposons and the retrocopies share stretches of microsimilarity, suggesting the occurrence of microsimilarity-mediated recombination; (2) the insertion sites are mostly genic, the preferential insertion site of LTR retrotransposons; and (3) these new loci show signs of acting as nonautonomous LTR retrotransposons, being subjected to further retroposition cycles.

\section{LTR retrotransposons mediate retroposition across animals}

Since Drosophila polymorphic retrocopies show the signatures of LTR-mediated retroposition, we also expect to find these features in recently originated retrocopies encoded by the $D$. melanogaster reference genome. By modifying the strategy in Bai et al. (2007) (Methods), we identified five retrocopies present in the reference genome but absent from the closely related D. simulans and D. sechellia (Table 3; Supplemental Data Set 7). The youngest,

Table 3. The five newly originated Drosophila retrocopies

\begin{tabular}{|c|c|c|c|c|c|c|c|}
\hline Parent/Retrocopy ${ }^{a}$ & $\begin{array}{l}\text { Retrocopy } \\
\text { location }\end{array}$ & $\begin{array}{c}\text { Parent/retrocopy } \\
\text { transcript } \\
\text { length (bp) }\end{array}$ & $\begin{array}{l}\text { Diverg- } \\
\text { ence }^{\mathrm{b}}\end{array}$ & $\begin{array}{c}\text { Microsi- } \\
\text { milarity (bp) }\end{array}$ & $\begin{array}{c}\text { Flanking transposon, } \\
\text { transposon family, } \\
\text { divergence }\end{array}$ & Poly(A) & TSDs \\
\hline CG33932/CG33932_r & $\begin{array}{l}\text { Uextra:5521091- } \\
5521902^{\mathrm{e}}\end{array}$ & $1244 / 812$ & $0.10 \%$ & $-11^{d}$ & MAX, Pao, $3.70 \%$ & - & - \\
\hline CG16728/CG16728_r & $\begin{array}{c}X: 17689090- \\
17689601\end{array}$ & $2489 / 512$ & $0.90 \%$ & 7,2 & $\begin{array}{l}\text { BLASTOPIA, Gypsy, } \\
0.20 \%\end{array}$ & - & - \\
\hline CG2033/CG12324 & $\begin{array}{c}2 \mathrm{R}: 6709256- \\
6709889\end{array}$ & $678 / 634$ & $2.30 \%$ & - & $\begin{array}{l}\text { No associated repeat } \\
\text { elements }\end{array}$ & AAAA & - \\
\hline CG1742/CR12628 & $\begin{array}{c}2 \mathrm{~L}: 22226151- \\
22226773\end{array}$ & $667 / 623$ & $3.50 \%$ & $-14,9$ & $\underset{10 \%{ }^{f}}{G 3 D D M}$ Jockey, LINE, & AAAAAAA & AATCA/AATCA \\
\hline CG3265/CG4035 $4^{\mathrm{g}}$ & $\begin{array}{l}\text { 3LHet:687408- } \\
\quad 688819\end{array}$ & $1873 / 1412$ & $4.00 \%$ & $2^{\mathrm{h}}$ & TV1, Gypsy, 27.50\% & - & $\begin{array}{l}\text { АТTCTTCTTG/ } \\
\text { АТСTTCTTG }\end{array}$ \\
\hline
\end{tabular}

${ }^{\mathrm{a} B}$ Because the function of these genes (except CG12324 or Rps15ab) is not characterized, we use the term retrocopy rather than retrogene.

${ }^{b}$ Divergence reflects the nucleotide differences between parental genes and retrocopies.

${ }^{\mathrm{C}}$ The average divergence relative to the consensus sequence generated by RepeatMasker.

${ }^{d}$ A sequencing gap flanks one end of the retrocopy preventing the identification of microsimilarity stretches.

${ }^{\mathrm{e}}$ The sequence of this retrocopy is absent from the new D. melanogaster Release 6 (Dm6). However, we could find exon-exon spanning reads in more than one DGRP line, suggesting that it is genuine.

${ }^{\mathrm{f}}$ The presence of a continuous Jockey element in the closely related species, D. simulans, suggests that the retrocopy was inserted into this preexisting element (Supplemental Fig. 11).

${ }^{9}$ CG40354 is annotated as a pseudogene in Dm6.

${ }^{\mathrm{h}}$ TV1 is immediately adjacent to this retrocopy at the $3^{\prime}$ flanking region, whereas the QUASIMODO Gypsy element is located in the $5^{\prime}$ flanking region 111 bp away (Supplemental Fig. 4B). So, only microsimilarity relative to TV1 is examined.

\section{Genome Research}

www.genome.org 
CG33932_r and CG16728_r , are flanked by LTR retrotransposons, and CG16728_r additionally has microsimilarities at both junctions. Consistently, no hallmarks of L1-mediated retroposition exist in the flanking regions of these two retrocopies (Supplemental Table 7). In contrast, the relatively older retrocopies, CG12324 and CR12628, are not flanked by LTR retrotransposons and are instead associated with candidate poly(A) tails and/ or TSDs (Toba and Aigaki 2000) suggesting a non-LTR-mediated retroposition. The final retrocopy (CG40354) is ambiguous because it harbors a 2-bp microsimilarity and a $3^{\prime}$ truncation (121 bp) but also candidate TSDs (Supplemental Fig. 4A; Supplemental Data Set 7). Since this locus situates in a repeatrich region that does not have a long synteny with closely related species (Supplemental Fig. 4B), we could not infer if it was formed through an LTR-mediated or non-LTR-mediated retroposition followed by secondary mutations.

However, no LTR retrotransposon remnants were found flanking 96 old retrogenes that predate the species split of D. melanogaster and D. simulans (Bai et al. 2007). Similarly, only three retrogenes (CG1924, CG13732, and CG5650) are associated with either candidate poly(A) tails or TSDs (Supplemental Table 7). The absence of the sequence features diagnostic of the type of retroposition event is expected for old retrogenes. It is a consequence of the rapid removal of flanking hallmark sequences by the strong deletion bias of D. melanogaster (Petrov and Hartl 1998) and by the accumulation of substitutions after the retroposition event.

Because LTR retrotransposons are widely shared across species (Havecker et al. 2004; Eickbush and Jamburuthugoda 2008), we searched additional animal genomes for signs of LTR-mediated retroposition. Specifically, we applied the pipeline used to identify recently originated retrocopies in Drosophila to five other species (mosquito, zebrafish, chicken, mouse, and human) representing a broad taxonomic breadth (Methods). We found retrocopies potentially generated by LTR retrotransposons in all species. In mosquito, zebrafish, and chicken we identified 3,7 , and 10 retrocopies, respectively, still retaining sequence features indicative of the underlying retroposition mechanism (Supplemental Table 8 ). We found that LTR retrotransposons are responsible for $\sim 50 \%$ of retrocopies (1/3 in mosquito, 5/7 in zebrafish, and $4 / 10$ in chicken). One example is the mosquito retrocopy ENSANGG00000011308, which is flanked by the LTR retrotransposon BEL18_AG. It is associated with 5- and 7-bp microsimilarity stretches at the switch points (Fig. 1C), an extensive 3 ' truncation $(\sim 1 \mathrm{~kb})$, and no signs of a poly(A) tail or TSDs (Supplemental Table 8).

In mammals, L1 retrotransposons are known to be a major driver of retroposition (Esnault et al. 2000; Kaessmann et al. 2009; Abyzov et al. 2013) and have generated thousands of retrocopies (Carelli et al. 2016). We implemented multiple filters (Methods) to identify the possibly small number of retrocopies created by LTR retrotransposons. We identified three retrocopies in human (including the previously described FAM8A1_r) (Jamain et al. 2001) and eight retrocopies in mouse that are flanked by LTR retrotransposon remnants and are not associated with any L1 hallmark sequences (Supplemental Table 8). The sequence structures of these retrocopies match those observed in Drosophila, mosquito, zebrafish, and chicken. For example, in mouse, a partial transcript (549/1646 bp) of ENSMUSG00000021752 was retroposed by the LTR retrotransposon IAPEy, mediated by 10 bp of microsimilarity and 2 bp of de novo sequence (Fig. 1D). In an exceptional example, we identified a "Super" retrocopy in which mRNAs derived from four distinct genes and one Alu element were co-retroposed into a single locus (Supplemental Table 8; Supplemental Fig. 5). Overall, despite the older age of the mouse retrocopies, we were still able to identify stretches of microsimilarity for nine of the 22 switch points. Unlike in Drosophila, where most retrocopies are located within genes (Table 2), in mouse, seven of the eight retrocopies are intergenic (Fisher's exact test $P=0.03$ ) (Supplemental Table 8), which is consistent with the well-known depletion of LTR retrotransposons within or in the vicinity of genes in mammals (Jern and Coffin 2008).

\section{LTR retrotransposons drive the initial transcription of retrocopies in both fly and mouse}

The prevalence of polymorphic retrocopies with more than one insertion site in Drosophila suggests that retrocopies mediated by LTR retrotransposons can be retroposed again as nonautonomous retrotransposons (Table 2). This also implies that the internal retrocopies and flanking retrotransposons are cotranscribed as chimeric transcripts by the regulatory sequences of the LTR retrotransposons. Hence, we tested whether the retroposed loci are transcribed as a single unit and whether the retrocopies share the transcriptional profile of the corresponding LTR retrotransposons. First, we performed RT-PCR on the 10 retrocopies for which we could design primers spanning the switch points (Supplemental Table 9). We detected the expression of all 10 retrocopies plus the flanking LTRs in both male and female adult bodies (Fig. 2A). In addition, both long-range RT-PCR for retrocopy CG5020_r and rapid amplification of cDNA ends (RACE) for CG17604_r , confirmed the transcription of the whole chimeric unit from the $5^{\prime}$ to the $3^{\prime}$ LTRs (Methods; Supplemental Data Sets 3,4$)$. Second, we profiled the expression of the 10 retrocopies and corresponding retrotransposons in testis, ovary, and head (Methods). We targeted these three organs because in Drosophila, a large fraction of retrogenes are testis-specific or testis-dominant (Supplemental Fig. 6; Betrán et al. 2002; Vibranovski et al. 2009), but LTR retrotransposons tend instead to be abundantly transcribed in the head (Perrat et al. 2013). The ovary was included for comparison. We found that nine of the 10 retrocopies show a strong positive correlation between the expression of the retrocopy and the corresponding LTR retrotransposon, with eight retrocopies showing their highest expression in the head, more than expected by chance (binomial test $P=0.02$ ) (Fig. 2B). RT-PCR further confirmed the expression in the head of four retrocopies encoded by line 208 (Fig. 2C). Interestingly, the expression intensity of LTR retrotransposons is generally higher than that of the corresponding retrocopies except for STALKER2 (CG5119_CR42443_r) and Gypsy5 (CG3631_r) (Fig. 2B), which could be explained by the fact that these two retrotransposons have a lower copy number in the genome relative to the others (1-3 versus 4-27, Wilcoxon rank test one-tailed $P=0.03$ ) (Supplemental Table 10). This also suggests that retrocopies as nonautonomous retrotransposons could be less repressed than autonomous elements (Maksakova et al. 2006).

The transcriptional characteristics of the mouse LTR-mediated retrocopies are similar to those of Drosophila with respect to both the transcription structure and expression profile. Specifically, we found Mouse ENCODE Consortium RNA-sequencing (RNA-seq) reads (Yue et al. 2014) spanning the switch points between the LTR retrotransposons and the retrocopies for four of the eight LTR-mediated retrocopies, suggesting that they are transcribed as a single transcript (Methods; Supplemental Table 11; 
A

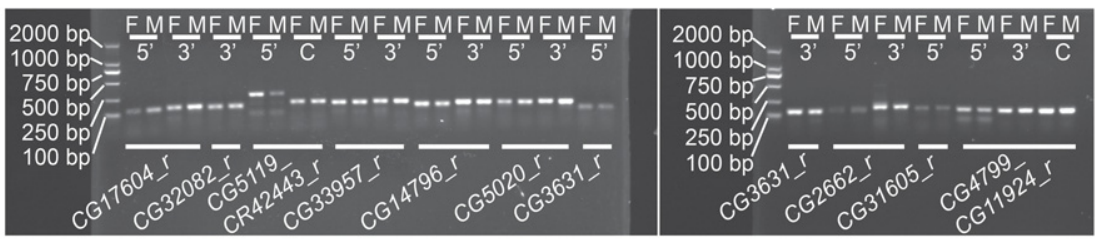

B

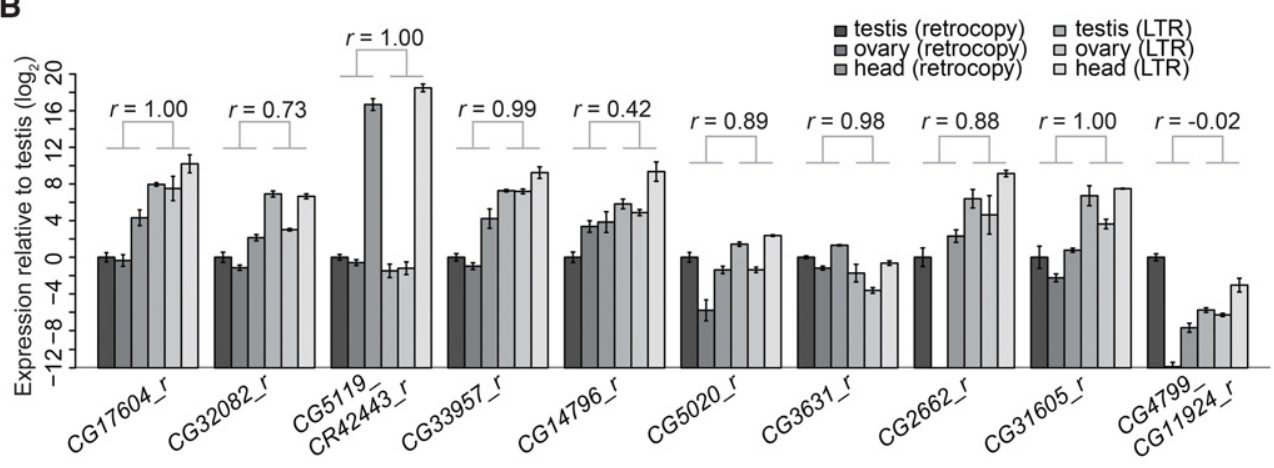

C
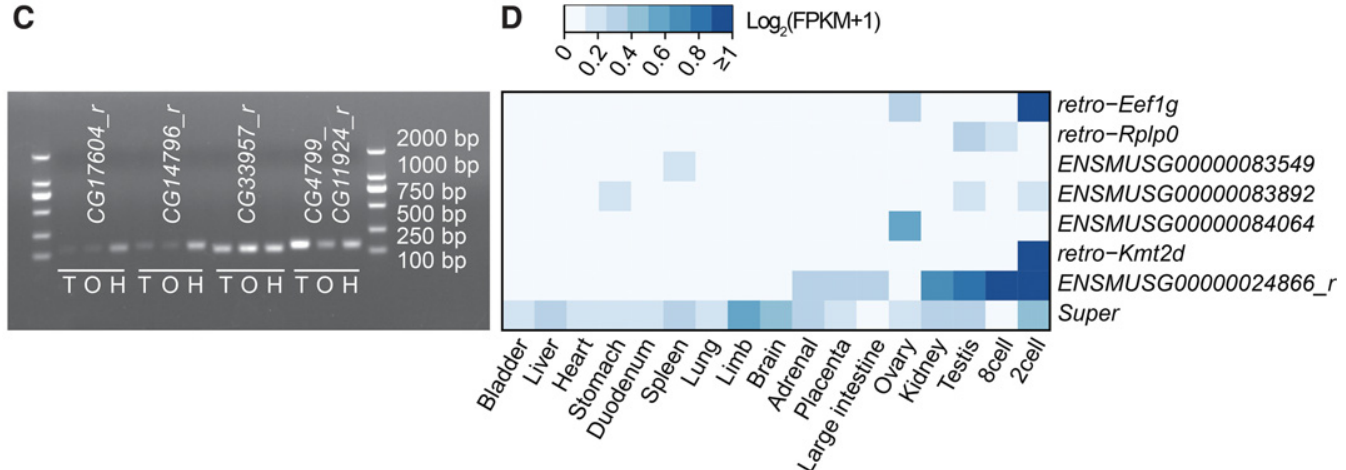

Figure 2. The expression of retrocopies in fruit fly and mouse. $(A)$ Whole-body RT-PCR results for 10 retrocopies validated in Supplemental Figure 2, which are expressed in both male $(\mathrm{M})$ and female $(F)$. The primer sequences and expected product sizes are listed in Supplemental Table 9 . For the chimeric genes, CG5119_CR42443_r and CG4799_CG11924_r, we also designed primers flanking the fusion point between the two parental genes, the product of which is marked with "C." (B) Tissue profiling via qRT-PCR. CG2662_r has no detectable expression in ovary. The Pearson correlation ( $r$ ) between the retrocopies and corresponding retrotransposons across the three tissues is displayed above. (C) Tissue level RT-PCR results for four of 10 retrocopies encoded by line 208 across Testis (T), Ovary (O), and Head (H). (D) Expression profile of retrocopies encoded by the mouse genome. Tissues are hierarchically clustered on the basis of expression similarity across genes. Retrocopies are not clustered but instead are sorted by age as approximated by nucleotide divergence. To reveal relative moderate expression, the color-code scheme is truncated at the FPKM cutoff of 1 (i.e., all expression higher than 1 is shown in dark blue).

Supplemental Fig. 7). In the case of the Super retrocopy, longrange RT-PCR experiments showed that the fragments derived from the four different parental genes are transcribed together, also suggesting the whole locus represents a single unit (Supplemental Fig. 8). Furthermore, unlike in the fly where LTR retrotransposons tend to be broadly transcribed (Supplemental Fig. 9), LTR retrotransposons in mouse are generally tightly controlled at the transcriptional level and tend to be tissue-specific with a strong bias toward early embryogenesis (Maksakova et al. 2006; Faulkner et al. 2009; Macfarlan et al. 2012). Consistently, four of the eight retrocopies show the highest expression at the twocell or eight-cell stages across 17 tissues/developmental stages, which is not expected by chance (binomial test $P=0.009$ ) (Fig. 2D; Supplemental Table 11). Actually, four could be an underestimation given that genes without high transcriptional levels (Fragments Per Kilobase of exon per Million fragments mapped, FPKM > 100) like LTR-mediated retrocopies (Supplemental Table 11) tend to be neglected in single-cell RNA-seq data
(Marinov et al. 2014). Overall, these lines of evidence in mouse again support the hypothesis that the expression of LTR-mediated retrocopies is driven by the LTR retrotransposons' regulatory sequences.

LTR-mediated retrocopies are more often transcribed from the same strand as the parental genes and capable of generating inframe proteins

Since LTRs (at the two ends) are known to have the potential to act as bidirectional promoters (Feuchter and Mager 1990; Dunn et al. 2006; Faulkner et al. 2009), it is unclear whether the transcription of the retrocopies occurs from the sense strand relative to the parental genes. In Drosophila, a RACE experiment demonstrated that CG17604_r is transcribed from the sense strand (Supplemental Data Set 4). In order to identify the direction of transcription for more retrocopies, we generated strand-specific RNA-seq data for testis, ovary, and head for line 208 (Methods; 
Supplemental Table 5; Supplemental Fig. 10). Given the high sequence identity between polymorphic retrocopies and their parental genes, we evaluated the strandedness using only the reads spanning the switch points. We did not find any spanning reads for CG17604_r and CG33957_r but we detected reads supporting the transcription of CG14796_r and CG4799_CG11924_r across all three tissues (Fig. 3A). Specifically, we found that CG14796_r is transcribed from the antisense strand in head, but from the sense strand in ovary. In agreement with the relatively low expression of CG14796_r in testis (Fig. 2B), we only found weak expression in this tissue. CG4799_CG11924_r is bidirectionally transcribed in ovary and head, and additionally shows very high levels of sense transcription in testis (Fragment count Per Million fragments mapped, FPM $=14,111$ ), a result also consistent with the qRTPCR result (Fig. 2B). Thus, the RACE and RNA-seq experiments showed that three of four retrocopies are capable of transcription from the sense strand relative to the parental genes.

Analogously, by taking advantage of public strand-specific RNA-seq data from mosquito, chicken, zebrafish, and mouse (Methods), we found that recently evolved retrocopies in these species are also transcribed more often or more strongly from the sense strand (relative to their parental genes). Overall, 12 of 18 retrocopies are moderately transcribed (FPKM $>0.1$ ) from the sense strand in at least one tissue, whereas only six reach the same intensity from the antisense strand (Fig. 3).
The transcription of the retrocopies from the same strand as the parental genes suggests the possibility that the retrocopies could lead to in-frame translation. In support of this idea, for 14 (82\%) of the 17 Drosophila polymorphic or recently evolved retrocopies mediated by LTR-retrotransposons, the predicted longest ORF is in-frame relative to the parental proteins (Supplemental Table 12) possibly because they are too young (nucleotide divergence $<1 \%$ ) (Table 3 ) to have accumulated loss-of-function mutations. For relatively older retrocopies in other animals (nucleotide divergence $\geq 1 \%$ ) (Supplemental Table 8), 14 of 21 have accumulated at least one loss-of-function mutation (Supplemental Data Set 8). We examined directly the level of constraint at the protein-level by calculating $K_{\mathrm{A}} / K_{\mathrm{S}}$ values (the ratio between the nonsynonymous and the synonymous substitution rates) for the longest ORF predicted relative to the parental protein. We found that ENSDARG00000076317_r from zebrafish and ENSGALG00000011896_r from chicken have a $K_{\mathrm{A}} / K_{\mathrm{S}}$ ratio of 0.145 and 0.239 , respectively, which is significantly lower than the conservative cutoff of 0.5 and suggestive of constraint $(P=$ 0.041 and 0.004) (Supplemental Table 13; Betrán et al. 2002). ENSANGG00000011308 from mosquito also reached a marginal significance $\left(K_{\mathrm{A}} / K_{\mathrm{S}}=0.261, P=0.075\right)$. Therefore, these retrocopies, together with genes like Bs1 from maize (Elrouby and Bureau 2001, 2010), suggest that a fraction of LTR-mediated retrocopies can encode functional proteins.

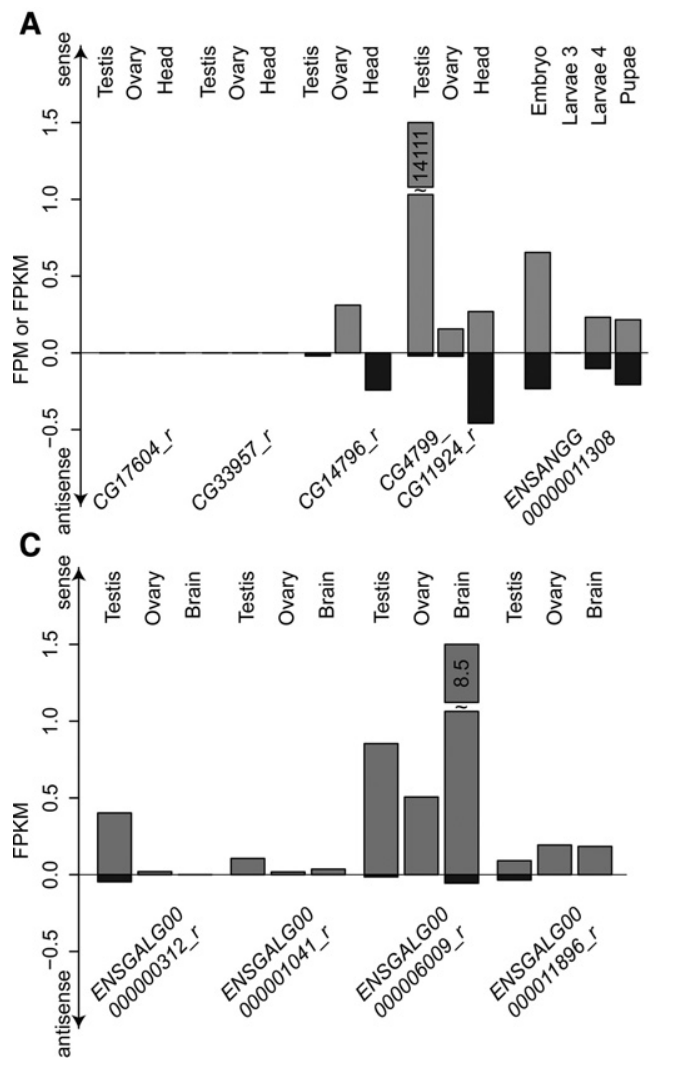

B

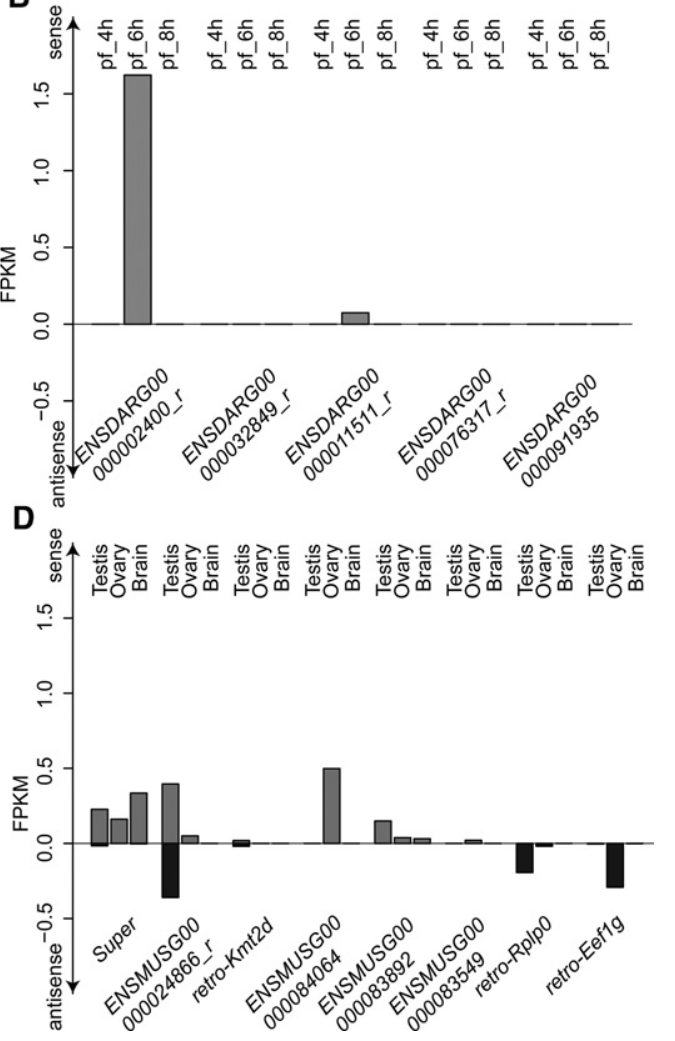

Figure 3. Strand-specific RNA-seq-based quantification. On the basis of newly generated and public strand-specific RNA-seq data, we quantified the expression of LTR-mediated retrocopies in fruit fly and mosquito $(A)$, zebrafish $(B)$, chicken $(C)$, and mouse $(D)$. For fruit fly, given the high similarity between polymorphic retrocopies and parental genes, only switch points spanning reads were counted, and FPM was calculated. For all other cases, FPKM was calculated. For mosquito, embryo, the third instar larvae, the fourth instar larvae, and pupae were profiled. For zebrafish, 4-, 6- and 8-h post fertilization (pf) stages were profiled. 


\section{Discussion}

\section{LTR-mediated retroposition is deeply conserved across eukaryotes}

By looking at young retrocopies in a broad range of taxa, we showed that retrogenes can be created in both invertebrates and vertebrates through an LTR-mediated retroposition mechanism. Our data suggest a model in which the RT starts synthesizing cDNA using the template from the RNA of the LTR retrotransposon, but induced by a stretch of microsimilarity between the LTR retrotransposon and a cellular mRNA captured within the VLP, the enzyme moves to the latter and takes the cellular mRNA as the template (Fig. 4). A second stretch of microsimilarity between the cellular mRNA and the LTR retrotransposon leads to another switch, this time back to the LTR retrotransposon to end the reverse transcription process.

This mechanism creates retrocopies with four unique features. First, because the stretches of microsimilarity can occur anywhere, mRNAs are often only partially duplicated (Table 1). Second, because only small stretches of microsimilarity are required, LTR-mediated retroposition can give rise to chimeras involving multiple parental genes. It can therefore be considered as a novel mechanism of gene recombination, including domainor exon-shuffling to capture the essence whereby the sequences of previously existing genes are combined as novel gene structures (Gilbert 1978; Moran et al. 1999; Graur and Li 2000). Third, a key feature of LTR-mediated retrocopies is their immediate transcription by the LTR's promoters. Fourth, LTR-mediated retrocopies can act as nonautonomous LTR retrotransposons and be subject to further duplication (Fig. 4).
This mechanism is compatible with the results from the limited studies on LTR-mediated retroposition in plants and yeast. In plants, although the retrocopies described are generally old (Elrouby and Bureau 2001, 2010; Wang et al. 2006), they show the typical chimeric structure with internal retrocopies flanked by retrotransposons. Specifically, in maize, the gene Bs1 combines the partial exonic sequences of three parental genes and is flanked by an LTR retrotransposon (Johns et al. 1985; Elrouby and Bureau 2001, 2010). The copy number of Bs1 ranges from one to five across various species of the genus Zea, and all individual copies share the same switch points (Elrouby and Bureau 2010). Thus, Bs1 likely has undergone secondary retropositions as seen for CG17604_r or CG10212_r (Table 2). In contrast to these efforts in plants and our work on animals, the work in yeast studied retroposition by inducing or screening artificial retrocopies via cellular assays (Derr et al. 1991; Schacherer et al. 2004; Maxwell and Curcio 2007). Still, these experiments recapitulated the unique features associated with LTR-mediated retroposition such as the presence of microsimilarity and the transcriptional capability (Schacherer et al. 2004; Maxwell and Curcio 2007).

Thus, if we combine our current work in animals with previous work on individual genes in plants and artificially induced systems in yeast, we see a striking consistence of the features that characterize LTR-mediate retroposition: chimeric structures between partial duplicates and LTR retrotransposons, microsimilarity at the junction points, the capacity for transcription, and the existence of secondary retroposition events (Fig. 4). Template-switch dependent LTR-mediated retroposition is therefore highly conserved across multiple eukaryotic lineages.

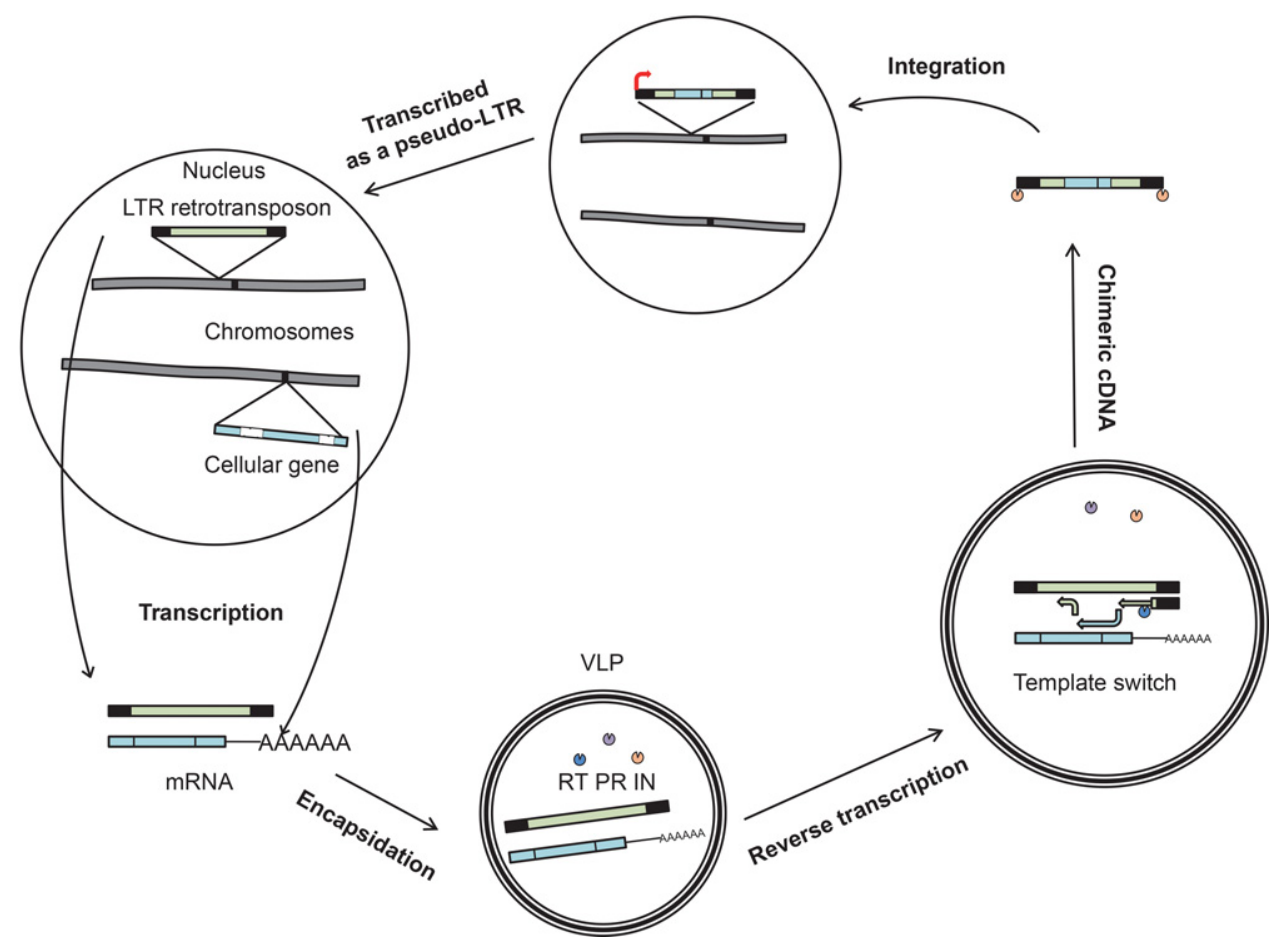

Figure 4. A schematic representation of the template switch model for LTR-mediated retroposition. (VLP) virus-like particle; (RT) reverse transcriptase; (PR) protease; (IN) integrase. The black boxes in LTR retrotransposons correspond to the LTRs and the light green box to the ORF. The blue boxes correspond to exons encoded by the host genome and the white boxes to introns. The double template switch occurs in the VLP, generating a chimeric CDNA (multiple switches can occur) (Derr et al. 1991; Schacherer et al. 2004; Maxwell and Curcio 2007). After integration, the chimeric sequences can be transcribed as pseudo-LTR retrotransposons, which could be subject to further cycles of retroposition (Elrouby and Bureau 2010).

\section{Genome Research}

www.genome.org 
Although LTR-mediated retroposition is active across different lineages, its contribution to the formation of retrogenes varies widely across taxa. Our previous work in plants has found that LTR- and non-LTR-mediated retroposition appear to occur with roughly equal rates in dicots (Arabidopsis thaliana and Manihot esculenta) (Zhu et al. 2016). In this work, we confirm that L1-mediated retroposition is the dominant mechanism of retroposition in human and mouse (Kaessmann et al. 2009). In mosquito, zebrafish, and chicken the contribution is more balanced with each contributing roughly equally to the formation of retrocopies. Although in Drosophila, we found similar numbers of recently originated retrocopies created by both mechanisms, all polymorphic retrocopies were associated with LTR-mediated events. Through simulations (Methods; Supplemental Table 14) we show that our pipeline works equally well detecting LTR-mediated and nonLTR-mediated retroposition. Therefore, the enrichment of LTRmediated retroposition among polymorphic retrocopies when compared to those recently originated (Fisher's exact test $P=$ 0.04 ) likely reflects the recent increase in activity of LTR retrotransposons in flies (Bergman and Bensasson 2007; Kofler et al. 2015).

\section{The different evolutionary trajectories of L1-mediated and LTR-mediated retrocopies}

L1-mediated retrocopies are long known to be mostly dead-on-arrival because of the absence of the parental genes' regulatory sequences (Mighell et al. 2000; Kaessmann et al. 2009). A recent work identified approximately 5000 retrocopies across various placental mammals and found that only $4 \%$ were robustly expressed $(F P K M \geq 1)$ (Carelli et al. 2016). The expressed retrocopies were found to have evolved their promoters de novo or to have recruited proto-promoters from nearby regions (Carelli et al. 2016). In our study, the two recently evolved non-LTR-mediated retrocopies in Drosophila also demonstrate that the fate of these retrocopies depends on whether a promoter is fortuitously gained. CG12324 is broadly transcribed, likely because it hitchhiked regulatory sequences already present at the insertion site, as suggested by the correlation between its expression and that of its neighboring gene, $C G 12323$ (Pearson $r=0.76, P=0.005$ ) (Supplemental Table 15). The other one, CR12628, is not transcribed, presumably because of the absence of regulatory sequences at the insertion site (Table 3; Supplemental Fig. 11). In contrast, we found that LTR-mediated retrocopies are transcribed immediately after the retroposition (Fig. 2). This phenomenon may be restricted to the fixation stage and to a short period after fixation. Consistently, we only detect reads spanning switch points for four retrocopies encoded by the mouse reference genome, suggesting that their coexpression may eventually get decoupled. More importantly, four retrocopies (Supplemental Table 8) encoded by zebrafish and chicken already lost the LTR retrotransposon remnants at one side, suggesting that LTR-mediated retrocopies eventually diverge from nonautonomous LTR retrotransposons.

\section{A significant proportion of LTR-mediated retrocopies may play novel functions}

Our work shows that LTR retrotransposons can create new transcribed retrocopies. Therefore, from the viewpoint of transcription, the potential of LTR-mediated retroposition to create functional retrocopies is higher than that of L1-mediated retroposition. But are any of these retrocopies actually functional (i.e., bona fide retrogenes)? Although the young age of the retrocopies makes it difficult to test the levels of protein constraint, we have identified two or three retrocopies showing evidence for constraint at the protein level (Supplemental Table 13).

We should note that translation does not absolutely require that the retrocopies are in the same frame as the parental genes. In the case of Bs1 in maize, the region derived from one parental gene is out-of-frame, whereas the regions derived from the other two parental genes are in-frame (Elrouby and Bureau 2001, 2010). Moreover, loss-of-function mutations can be rescued by the evolution of new introns within the retrocopies (Tan et al. 2014). In addition, evidence has accumulated suggesting the general significance of long noncoding RNAs (lncRNA) (Ponting et al. 2009; Guttman and Rinn 2012; Rinn and Chang 2012). The Drosophila retrocopy, CG4799_CG11924_r, is more highly expressed than $99.5 \%$ of the genes transcribed in testis (Supplemental Fig. 12) and has therefore the potential to act as a functional lncRNA if it could not encode a protein. Consistently, we detected reduced levels of nucleotide diversity and increased levels of linkage disequilibrium (LD) around this locus, suggesting that positive selection could be driving its spread (Supplemental Fig. 13).

If some LTR-mediated recopies are indeed functional, their chimeric structure implies that they should have a radically different functional role compared to their parental genes. The most extreme examples are those of retrocopies involving several parental genes. Bs1 in maize shows that these complex chimeric genes can indeed sometimes become functional hybrid proteins (Jin and Bennetzen 1994; Elrouby and Bureau 2001, 2010). Therefore, it is reasonable to conclude that LTR retrotransposons are capable of creating retrocopies across a wide range of eukaryotes, which could subsequently evolve to be neofunctionalized retrogenes given their novel gene structure and expression profile.

\section{Methods}

\section{Public Drosophila population resequencing data}

We focused on the subset of 40 inbred lines from the DGRP project (NCBI SRA database: SRP000694 and SRP000224), which were also sequenced by the Drosophila Population Genomics Project (DPGP) project (Langley et al. 2012).

\section{Terminology}

Traditionally, "retrocopy" is used to refer to an intronless copy derived from a host mRNA. This terminology was developed for L1mediated retrocopies, which generally have no additional flanking sequences. However, for LTR-mediated retroposition, the retroposed locus consists not only of the sequence derived from the host mRNA but also of the flanking LTR retrotransposon sequences. To be consistent with previous studies, we use the term retrocopy to refer only to the host mRNA-derived sequence. We refer to retrocopies using the ID of the parental gene followed by "_rn" except when the gene is already annotated.

\section{Detection of Drosophila polymorphic retrocopies}

To create the search library, we extracted exon-exon junction sequences by pooling $2 \times 100 \mathrm{bp}$ from each pair of consecutive exons (Supplemental Fig. 1A). All reads were mapped to this library with Novoalign (http://www.novocraft.com), and only uniquely mapping reads were retained (Supplemental Methods). We called candidate retroposition or intron loss events if there were at least two reads spanning one exon-exon junction. Since the introns of parental genes are not deleted by retroposition, we retained 31 
candidates with reads mapping to both exon-intron and intronexon junctions (Supplemental Fig. 1B).

\section{Local assembly and validation of the polymorphic retrocopies}

For each candidate retrocopy, we extracted the reads that mapped uniquely to the parental genes, 500-bp flanking regions, and to exon-exon junctions and performed de novo local assembly with MIRA (Supplemental Fig. 1C; Chevreux et al. 1999). MIRA will export at least two different contigs corresponding to the parental and retrocopy locus (Supplemental Fig. 1D). If the contig encoded by the retrocopy was too short to identify its exact switch points, it was further assembled with PRICE (Ruby et al. 2013). We assembled 15 retrocopies derived from 17 parental genes as well as their flanking regions (10-200 bp) (Supplemental Data Set 1). We validated this data set by PCR (Schrider et al. 2011, 2013) in which we used Primer3 (Untergasser et al. 2012) to design primers targeting the exons shared by parental genes and retrocopies that flank at least one intron in the parental genes (Supplemental Table 4). If retrocopies are present, we should amplify two PCR products of different sizes, the larger corresponding to the parental gene (introns present) and the smaller to the retrocopy (introns absent).

\section{Evaluation of potential bias in the detection of LTR-mediated and non-LTR-mediated retrocopies}

We ran simulations approximating the process of retroposition in Drosophila. Because there are more full-length LTR retrotransposons in this species (Supplemental Table 16), we randomly selected 105 genes out of the top 600 most highly transcribed genes in testis and ovary as the parental genes to simulate 90 retrocopies mediated by LTR retrotransposons and 15 retrocopies mediated by nonLTR (L1) retrotransposons (Supplemental Table 14). We determined the size of the retroposed sequences, of the replaced regions of the LTR retrotransposons, and of the microsimilarities following the distributions learned from the empirical set of 15 retrocopies. We simulated non-LTR-mediated retrocopies with $5^{\prime}$ truncation ( $\leq 500 \mathrm{bp}$ ), poly(A) tails ( $\leq 100 \mathrm{bp})$, and TSD ( $\leq 10 \mathrm{bp}$ ) based on previous studies (Linheiro and Bergman 2012; Subtelny et al. 2014). We randomly inserted the simulated retrocopies into intergenic or intronic regions. Finally, we simulated sequencing reads with a coverage of $50 \times$ and with variable read length as in the DGRP data (Supplemental Table 14). We were able to recover 85/90 simulated LTR-mediated retrocopies and 15/15 simulated L1-mediated retrocopies (Supplemental Table 14; Supplemental Methods), demonstrating that our pipeline is not biased in the detection of LTR-mediated and non-LTR-mediated retrocopies.

\section{Estimation of the nonrandomness of the microsimilarity length}

To test whether the extent of microsimilarity could have occurred purely by chance, we performed simulations for all 11 Drosophila retrocopies consisting of only two rounds of template switches. For each retrocopy, we randomly selected switch points. We selected the size of the retroposed sequences and of the replaced regions of the LTR retrotransposons following the normal distribution learned from our empirical set of retrocopies. For each retrocopy, we generated 100 random samples and recorded the size of the microsimilarity observed at both switch points. Supplemental Figure 3 shows the distribution of the microsimilarity length at both ends across 100 simulated events. The observed length (the red dot) is an outlier $(P<0.01)$ for 10 retrocopies. The exception is CG8567_r but even for this retrocopy, the total length of microsimilarity was only smaller or equal to the value observed in eight of the 100 simulations $(P=0.08)$. Overall, these data show that the extent of microsimilarity observed between the retrocopies and the LTR retrotransposons cannot be explained by chance.

\section{Inference of the retrocopies' insertion sites}

In order to identify the insertion sites, we generated wholegenome sequencing data using longer reads $(2 \times 250 \mathrm{bp})$ for six lines (e.g., 208) and mate-pair sequencing data for line 437 (Supplemental Table 5). We detected heterozygosity in these sequencing data that suggested contamination (admixture) between the lines sequenced. Thus in all analyses, these data were treated as pooled data. To confirm that the contamination occurred exclusively during the production of the novel sequencing data, we selected three loci with high SNP density and performed PCR experiments that confirmed that our fly stocks were not contaminated, showing the expected levels of homozygosity (Supplemental Fig. 14). With these whole-genome sequencing data, we were able to extend the flanking regions of the assembled retrocopies from a median of $163 \mathrm{bp}$ to $210 \mathrm{bp}$ and to locate four retrocopies. To identify the genomic insertion sites of more retrocopies, we further performed targeted high-throughput sequencing following the mobile element scanning (ME-Scan) protocol (Witherspoon et al. 2010) and located nine retrocopies (Table 2). Then, by PCR and Sanger sequencing, we validated 12 insertion sites for six retrocopies (Table 2; Supplemental Table 6).

We cloned and sequenced the two terminals of five retroposed loci and found that they include LTRs that are required for their integration into the genome (Supplemental Data Sets 3-6; Supplemental Methods).

\section{Identification of retrocopies in reference genomes}

In order to detect recently originated retrocopies in the reference genome of $D$. melanogaster, we refined a previous strategy (Bai et al. 2007) and searched the exon-exon junction library against the reference genome with BLASTN (Altschul et al. 1990). We retained only uniquely mapped regions and identified five $D$. melanogaster-specific retrocopies. Similarly, we scanned five other species: mosquito (UCSC assembly anoGam1), zebrafish (danRer7), chicken (galGal4), mouse (mm9), and human (hg19). In mouse, we found 208 retrocopies flanked by LTR retrotransposons on both sides. We excluded retrocopies inserted into preexisting LTR retrotransposons by implementing four filters: (1) The flanking LTR retrotransposons have to belong to the same LTR retrotransposon type or the nonautonomous retrotransposon mated with autonomous retrotransposon (ORR1/MaLR with MERVL, ETn with MusD) (McCarthy and McDonald 2004); (2) both the internal mRNAs and the flanking LTR retrotransposons have to be absent from the rat genome (rn4); (3) the LTR retrotransposons have to be discontinuous with a gap $>20 \mathrm{bp}$; and (4) there are no L1 hallmarks. Analogously, we filtered 518 human retrocopies flanked by LTR retrotransposons with the whole retroposed locus being required to be absent in the nonprimate placental mammals. In Supplemental Table 8, only the retrocopies that passed these filters are shown for mouse and human.

To approximate the evolutionary age, we calculated the overall nucleotide divergence between parental gene and retrocopy via BLAT (Kent 2002) for young retrocopies. For old retrogenes reported in Bai et al. (2007), we directly downloaded the synonymous substitution rate $\left(K_{\mathrm{S}}\right)$ values.

\section{Analysis of hallmark sequences of L1-mediated retroposition}

We called L1-mediated retrocopies by the presence of one of the following three features: (1) a poly(A) tail at the $3^{\prime}$ end; (2) TSDs at both ends; and (3) bias favoring truncation of $5^{\prime}$ rather than $3^{\prime}$

\section{Genome Research}

www.genome.org 
ends. The presence of a TTTT/AA endonuclease cleavage site has been shown to not be reliable (Richardson et al. 2014). We inferred the boundary between retrocopies and flanking regions by BLAT. In order to generate a conservative data set of LTR-mediated retrocopies, we specified relatively loose cutoffs for L1 hallmark sequences: a minimum of three continuous As in a 5-bp-long sequence at the $3^{\prime}$ end, and a minimal TSD not $<5$ bp with an identity $\geq 80 \%$. When retrocopies flanked by LTR retrotransposons were also associated with candidate hallmark sequences for L1-mediated retroposition, we determined whether the poly(A) tail and TSDs were part of the LTR retrotransposons and whether the LTR retrotransposons were discontinuous given the canonical annotations in Repbase (Kaminker et al. 2002).

\section{Transcriptional profiling of Drosophila polymorphic retrocopies}

We investigated the expression profile of polymorphic retrocopies using RT-PCR, qRT-PCR, RACE, and RNA-seq (Supplemental Methods). For LTR retrotransposons, we designed primers on the basis of the canonical sequence (Supplemental Table 9). To determine the internal controls in qRT-PCR, we followed the protocol in Gan et al. (2013) and chose the three most transcriptionally constant genes (eIF-1A, Rap2l, and SdhA) out of 26 in Ling and Salvaterra (2011) and Ponton et al. (2011). Strand-specific RNAseq data for line 208 were generated by following the dUTP protocol (Levin et al. 2010). Reads were mapped following a Novoalignbased RNA-seq pipeline, with the 15 retrocopies added to the reference sequence set. We only kept the reads spanning the switch points.

Transcriptional profiling of recently originated retrocopies across various animals

The FPKM data of the 96 Drosophila retrogenes were downloaded from FlyBase (Marygold et al. 2013). Only the genes CG5150 and CG9518 were absent from this data set. To investigate the expression profiles of the three older Drosophila retrocopies and of eight mouse retrocopies, we downloaded the FASTQ-format unstranded modENCODE RNA-seq data (Celniker et al. 2009) and stranded ENCODE mouse transcriptome data (http://hgdownload.cse.ucsc .edu/goldenPath/mm9/encodeDCC/wgEncodeCshlLongRnaSeq/). Since LTR retrotransposons are highly active in early mouse embryogenesis (Macfarlan et al. 2012), we also downloaded the single-cell RNA-seq data for two-cell and eight-cell stages from the NCBI GEO database (GSE44183). To better represent the transcriptome diversity, 12 representative fly tissues and 15 mouse tissues were chosen according to a tissue-level clustering analysis (Supplemental Fig. 15). Because retrocopies are highly similar ( $\geq 90 \%$ identity) to parental genes, we used only uniquely mapping reads and calculated the effective length of the retrocopy defined in Zhang et al. (2011) when calculating the FPKM values.

Furthermore, we analyzed public strand-specific data sets from mosquito (ArrayExpress ERP006838), zebrafish (Lee et al. 2013), and chicken (Necsulea et al. 2014). We estimated the quality of strandedness based on exon-exon junction spanning reads. Overall, all data sets are reasonably accurate, with $>89 \%$ reads (Supplemental Fig. 10) supporting the standard splicing site "GT-AG."

In order to determine whether a gene is called as present, we specified two FPKM cutoffs. Because it has been shown that FPKM values from 0.1 to 0.4 could reflect active transcription (Hart et al. 2013), we took the high cutoff of 1 to define robust expression as done in Carelli et al. (2016) and the low cutoff of 0.1 to define modest expression. For read counts, a cutoff of 0.1 (FPKM) corresponds to at least nine reads in Figure 3.

\section{Evolutionary analyses}

To examine protein-level constraints, we performed $K_{\mathrm{A}} / K_{\mathrm{S}}$ tests between the parental genes and corresponding retrocopies. By following Betrán et al. (2002), we tested whether $K_{\mathrm{A}} / K_{\mathrm{S}}$ is significantly smaller than 0.5 , which suggests functionality for both genes. First, we translated retrocopies by aligning parental proteins against retrocopies with exonerate (Slater and Birney 2005). Then, we aligned the proteins (for retrocopies with candidate loss-of-function mutations, the longest continuous peptide was used) with MAFFT (Katoh and Toh 2008) and then converted protein-level alignments into codon-level alignments via PAL2NAL (Suyama et al. 2006). Finally, we performed likelihood ratio tests via the codeml program in the PAML package (Yang 2007).

We tested whether CG4799_CG11924_r is under positive selection following Cardoso-Moreira et al. (2016). All SNPs located within $50 \mathrm{~kb}$ upstream of and downstream from 3L: 21,967,546 (the insertion site) (Table 2) were extracted from the DGRP variant annotation file (VCF) using VCFtools (Danecek et al. 2011). We then calculate levels of LD (as measured by $r^{2}$ ) and estimated diversity levels using VCFtools (Danecek et al. 2011).

\section{Data access}

The sequence data generated for this study have been submitted to the NCBI BioProject database (http://www.ncbi.nlm.nih. gov/bioproject/) under accession numbers PRJNA282433, PRJNA285001, PRJNA325420, and PRJNA325579, and Genome Sequence Archive (GSA; http://gsa.big.ac.cn/) under accession numbers PRJCA000123, PRJCA000124, PRJCA000279, and PRJCA000280 (Supplemental Table 5). Sanger trace files have been submitted to the NCBI Trace Archives (http://www.ncbi. nlm.nih.gov/Traces/home/index.cgi) with TI numbers in 2344 039996-2344040077.

\section{Acknowledgments}

We would like to thank three reviewers for their comprehensive and insightful comments. We thank the DGRP and DPGP groups for sharing the genomic data. We thank Bin He, Yang Shen, and Zhang laboratory members for helpful discussions. We also thank Wanzhu Jin for providing mouse samples. This research was supported by grants from the Strategic Priority Research Program of the Chinese Academy of Sciences (XDB13010400), the National Key Basic Research Program of China (Ministry of Science and Technology) (2015CB943001, 2013CB531202), the National Natural Science Foundation of China (91331114, 31322050), and the 1000 Young Talents Program to Y.E.Z. M.C.M. was supported by a Marie Curie incoming postdoctoral fellowship. Computing was jointly supported by the HPC Platform in BIG, CAS, and by HPC Platform, Scientific Information Center, Institute of Zoology, CAS, China.

Author contributions: Y.E.Z. conceived and designed the projects. S.T., C.C., Y.S., and L.L. performed computational analyses. S.T., W.S., D.Z., J.H., Y.M., H.J., Y.Z., Z.L., and X.H. performed the experiments. S.T., Y.E.Z., M.C.M., and M.L. analyzed the data. Y.E.Z., S.T., M.C.M., and M.L. wrote the paper.

\section{References}

Abyzov A, Iskow R, Gokcumen O, Radke DW, Balasubramanian S, Pei B, Habegger L, The 1000 Genomes Project Consortium, Lee C, Gerstein 
M. 2013. Analysis of variable retroduplications in human populations suggests coupling of retrotransposition to cell division. Genome Res 23: 2042-2052.

Altschul SF, Gish W, Miller W, Myers EW, Lipman DJ. 1990. Basic local alignment search tool. J Mol Biol 215: 403-410.

Bai Y, Casola C, Feschotte C, Betrán E. 2007. Comparative genomics reveals a constant rate of origination and convergent acquisition of functional retrogenes in Drosophila. Genome Biol 8: R11.

Bergman CM, Bensasson D. 2007. Recent LTR retrotransposon insertion contrasts with waves of non-LTR insertion since speciation in Drosophila melanogaster. Proc Natl Acad Sci 104: 11340-11345.

Betrán E, Thornton K, Long M. 2002. Retroposed new genes out of the X in Drosophila. Genome Res 12: 1854-1859.

Brosius J. 1991. Retroposons-seeds of evolution. Science 251: 753

Cardoso-Moreira M, Arguello JR, Gottipati S, Harshman LG, Grenier JK, Clark AG. 2016. Evidence for the fixation of gene duplications by positive selection in Drosophila. Genome Res 26: 787-798.

Carelli FN, Hayakawa T, Go Y, Imai H, Warnefors M, Kaessmann H. 2016. The life history of retrocopies illuminates the evolution of new mammalian genes. Genome Res 26: 301-314.

Celniker SE, Dillon LA, Gerstein MB, Gunsalus KC, Henikoff S, Karpen GH, Kellis M, Lai EC, Lieb JD, MacAlpine DM, et al. 2009. Unlocking the secrets of the genome. Nature 459: 927-930.

Chen S, Krinsky BH, Long M. 2013. New genes as drivers of phenotypic evolution. Nat Rev Genet 14: 744-744.

Chevreux B, Wetter T, Suhai S. 1999. Genome Sequence Assembly Using Trace Signals and Additional Sequence Information. In German Conference on Bioinformatics, pp. 45-56. Hannover, Germany.

Danecek P, Auton A, Abecasis G, Albers CA, Banks E, DePristo MA Handsaker RE, Lunter G, Marth GT, Sherry ST, et al. 2011. The variant call format and VCFtools. Bioinformatics 27: 2156-2158.

Delviks-Frankenberry K, Galli A, Nikolaitchik O, Mens H, Pathak VK, Hu WS. 2011. Mechanisms and factors that influence high frequency retroviral recombination. Viruses 3: 1650-1680.

Derr LK, Strathern JN, Garfinkel DJ. 1991. RNA-mediated recombination in S. cerevisiae. Cell 67: 355-364.

Dunn CA, Romanish MT, Gutierrez LE, van de Lagemaat LN, Mager DL. 2006. Transcription of two human genes from a bidirectional endogenous retrovirus promoter. Gene 366: 335-342.

Eickbush T. 1999. Exon shuffling in retrospect. Science 283: 1465-1467.

Eickbush TH, Jamburuthugoda VK. 2008. The diversity of retrotransposons and the properties of their reverse transcriptases. Virus Res 134: 221-234.

Elrouby N, Bureau TE. 2001. A novel hybrid open reading frame formed by multiple cellular gene transductions by a plant long terminal repeat retroelement. J Biol Chem 276: 41963-41968.

Elrouby N, Bureau TE. 2010. Bs1, a new chimeric gene formed by retrotransposon-mediated exon shuffling in maize. Plant Physiol 153: 1413-1424.

Engelman A, Oztop I, Vandegraaff N, Raghavendra NK. 2009. Quantitative analysis of HIV-1 preintegration complexes. Methods 47: 283-290.

Esnault C, Maestre J, Heidmann T. 2000. Human LINE retrotransposons generate processed pseudogenes. Nat Genet 24: 363-367.

Ewing AD, Ballinger TJ, Earl D, Broad Institute Genome Sequencing and Analysis Program and Platform, Harris CC, Ding L, Wilson RK, Haussler D. 2013. Retrotransposition of gene transcripts leads to structural variation in mammalian genomes. Genome Biol 14: R22.

Faulkner GJ, Kimura Y, Daub CO, Wani S, Plessy C, Irvine KM, Schroder K, Cloonan N, Steptoe AL, Lassmann T, et al. 2009. The regulated retrotransposon transcriptome of mammalian cells. Nat Genet 41: 563-571.

Feuchter A, Mager D. 1990. Functional heterogeneity of a large family of human LTR-like promoters and enhancers. Nucleic Acids Res 18: 1261-1270.

Gan H, Wen L, Liao S, Lin X, Ma T, Liu J, Song CX, Wang M, He C, Han C, et al. 2013. Dynamics of 5-hydroxymethylcytosine during mouse spermatogenesis. Nat Commun 4: 1995.

Ganko EW, Greene CS, Lewis JA, Bhattacharjee V, McDonald JF. 2006. LTR retrotransposon-gene associations in Drosophila melanogaster. J Mol Evo 62: $111-120$.

Gilbert W. 1978. Why genes in pieces? Nature 271: 501.

Goodrich DW, Duesberg PH. 1990. Retroviral recombination during reverse transcription. Proc Natl Acad Sci 87: 2052-2056.

Graur D, Li WH. 2000. Fundamentals of molecular evolution. Sinauer Associates, Sunderland, MA.

Guttman M, Rinn JL. 2012. Modular regulatory principles of large non-coding RNAs. Nature 482: 339-346.

Hajjar AM, Linial ML. 1993. A model system for nonhomologous recombination between retroviral and cellular RNA. J Virol 67: 3845-3853.

Hart T, Komori H, LaMere S, Podshivalova K, Salomon D. 2013. Finding the active genes in deep RNA-seq gene expression studies. BMC Genomics 14: 778 .
Havecker ER, Gao X, Voytas DF. 2004. The diversity of LTR retrotransposons. Genome Biol 5: 225.

Hindmarsh P, Leis J. 1999. Retroviral DNA integration. Microbiol Mol Biol Rev 63: $836-843$.

Jamain S, Girondot M, Leroy P, Clergue M, Quach H, Fellous M, Bourgeron T. 2001. Transduction of the human gene FAM $8 A 1$ by endogenous retrovirus during primate evolution. Genomics 78: 38-45.

Jern P, Coffin JM. 2008. Effects of retroviruses on host genome function. Annu Rev Genet 42: 709-732.

Jin YK, Bennetzen JL. 1994. Integration and nonrandom mutation of a plasma membrane proton ATPase gene fragment within the Bs1 retroelement of maize. Plant Cell 6: 1177-1186.

Johns MA, Mottinger J, Freeling M. 1985. A low copy number, copia-like transposon in maize. EMBO J 4: 1093-1101.

Kaessmann H, Vinckenbosch N, Long M. 2009. RNA-based gene duplication: mechanistic and evolutionary insights. Nat Rev Genet 10: 19-31.

Kaminker JS, Bergman CM, Kronmiller B, Carlson J, Svirskas R, Patel S, Frise E, Wheeler DA, Lewis SE, Rubin GM, et al. 2002. The transposable elements of the Drosophila melanogaster euchromatin: a genomics perspective. Genome Biol 3: RESEARCH0084.

Katoh K, Toh H. 2008. Recent developments in the MAFFT multiple sequence alignment program. Brief Bioinformatics 9: 286-298.

Kent WJ. 2002. BLAT-the BLAST-like alignment tool. Genome Res 12: 656-664.

Kofler R, Nolte V, Schlötterer C. 2015. Tempo and mode of transposable element activity in Drosophila. PLoS Genet 11: e1005406.

Langley CH, Stevens K, Cardeno C, Lee YC, Schrider DR, Pool JE, Langley SA, Suarez C, Corbett-Detig RB, Kolaczkowski B, et al. 2012. Genomic variation in natural populations of Drosophila melanogaster. Genetics 192: 533-598.

Lee MT, Bonneau AR, Takacs CM, Bazzini AA, DiVito KR, Fleming ES, Giraldez AJ. 2013. Nanog, Pou5f1 and SoxB1 activate zygotic gene expression during the maternal-to-zygotic transition. Nature 503: 360-364.

Levin JZ, Yassour M, Adiconis X, Nusbaum C, Thompson DA, Friedman N, Gnirke A, Regev A. 2010. Comprehensive comparative analysis of strand-specific RNA sequencing methods. Nat Methods 7: 709-715.

Li WH. 1997. Molecular evolution. Sinauer Associates, Sunderland, MA.

Ling D, Salvaterra PM. 2011. Robust RT-qPCR data normalization: validation and selection of internal reference genes during post-experimental data analysis. PLoS One 6: e17762.

Linheiro RS, Bergman CM. 2012. Whole genome resequencing reveals natural target site preferences of transposable elements in Drosophila melanogaster. PLoS One 7: e30008.

Long M, Rosenberg C, Gilbert W. 1995. Intron phase correlations and the evolution of the intron/exon structure of genes. Proc Natl Acad Sci 92: 12495-12499.

Luo GX, Taylor J. 1990. Template switching by reverse transcriptase during DNA synthesis. I Virol 64: 4321-4328.

Macfarlan TS, Gifford WD, Driscoll S, Lettieri K, Rowe HM, Bonanomi D, Firth A, Singer O, Trono D, Pfaff SL. 2012. Embryonic stem cell potency fluctuates with endogenous retrovirus activity. Nature 487: 57-63.

Maksakova IA, Romanish MT, Gagnier L, Dunn CA, van de Lagemaat LN, Mager DL. 2006. Retroviral elements and their hosts: insertional mutagenesis in the mouse germ line. PLoS Genet 2: e2.

Marinov GK, Williams BA, McCue K, Schroth GP, Gertz J, Myers RM, Wold BJ. 2014. From single-cell to cell-pool transcriptomes: stochasticity in gene expression and RNA splicing. Genome Res 24: 496-510.

Marygold SJ, Leyland PC, Seal RL, Goodman JL, Thurmond J, Strelets VB, Wilson RJ, FlyBase Consortium. 2013. FlyBase: improvements to the bibliography. Nucleic Acids Res 41: D751-D757.

Maxwell PH, Curcio MJ. 2007. Retrosequence formation restructures the yeast genome. Genes Dev 21: 3308-3318.

McCarthy EM, McDonald JF. 2004. Long terminal repeat retrotransposons of Mus musculus. Genome Biol 5: R14.

Mighell AJ, Smith NR, Robinson PA, Markham AF. 2000. Vertebrate pseudogenes. FEBS Lett 468: 109-114.

Moran JV, DeBerardinis RJ, Kazazian HH. 1999. Exon shuffling by L1 retrotransposition. Science 283: $1530-1534$.

Necsulea A, Soumillon M, Warnefors M, Liechti A, Daish T, Zeller U, Baker JC, Grützner F, Kaessmann H. 2014. The evolution of lncRNA repertoires and expression patterns in tetrapods. Nature 505: 635-640.

Parker HG, VonHoldt BM, Quignon P, Margulies EH, Shao S, Mosher DS, Spady TC, Elkahloun A, Cargill M, Jones PG, et al. 2009. An expressed fof 4 retrogene is associated with breed-defining chondrodysplasia in domestic dogs. Science 325: 995-998.

Perrat PN, DasGupta S, Wang J, Theurkauf W, Weng Z, Rosbash M, Waddell S. 2013. Transposition-driven genomic heterogeneity in the Drosophila brain. Science 340: 91-95.

Petrov DA, Hartl DL. 1998. High rate of DNA loss in the Drosophila melanogaster and Drosophila virilis species groups. Mol Biol Evol 15: 293-302.

\section{Genome Research}

www.genome.org 
Ponting CP, Oliver PL, Reik W. 2009. Evolution and functions of long noncoding RNAs. Cell 136: 629-641.

Ponton F, Chapuis MP, Pernice M, Sword GA, Simpson SJ. 2011. Evaluation of potential reference genes for reverse transcription-qPCR studies of physiological responses in Drosophila melanogaster. J Insect Physiol 57: 840-850.

Richardson SR, Morell S, Faulkner GJ. 2014. L1 retrotransposons and somatic mosaicism in the brain. Annu Rev Genet 48: 1-27.

Rinn JL, Chang HY. 2012. Genome regulation by long noncoding RNAs. Annu Rev Biochem 81: 145-166.

Ruby JG, Bellare P, Derisi JL. 2013. PRICE: software for the targeted assembly of components of (meta) genomic sequence data. G3 (Bethesda) 3: 865-880.

Schacherer J, Tourrette Y, Souciet JL, Potier S, De Montigny J. 2004. Recovery of a function involving gene duplication by retroposition in Saccharomyces cerevisiae. Genome Res 14: 1291-1297.

Schrider DR, Stevens K, Cardeno CM, Langley CH, Hahn MW. 2011. Genome-wide analysis of retrogene polymorphisms in Drosophila melanogaster. Genome Res 21: 2087-2095.

Schrider DR, Navarro FC, Galante PA, Parmigiani RB, Camargo AA, Hahn MW, de Souza SJ. 2013. Gene copy-number polymorphism caused by retrotransposition in humans. PLoS Genet 9: e1003242.

Slater GS, Birney E. 2005. Automated generation of heuristics for biological sequence comparison. BMC Bioinformatics 6: 31 .

Soares MB, Schon E, Henderson A, Karathanasis SK, Cate R, Zeitlin S, Chirgwin J, Efstratiadis A. 1985. RNA-mediated gene duplication: The rat preproinsulin I gene is a functional retroposon. Mol Cell Biol 5: 2090-2103.

Subtelny AO, Eichhorn SW, Chen GR, Sive H, Bartel DP. 2014. Poly(A)-tail profiling reveals an embryonic switch in translational control. Nature 508: $66-71$.

Suyama M, Torrents D, Bork P. 2006. PAL2NAL: robust conversion of protein sequence alignments into the corresponding codon alignments. Nucleic Acids Res 34: W609-W612.

Swain A, Coffin JM. 1992. Mechanism of transduction by retroviruses. Science 255: 841-845.

Tan S, Zhu Z, Zhu T, Te R, Zhang YE. 2014. Chance and necessity: emerging introns in intronless retrogenes. eLS doi: 10.1002/9780470015902. a0022886.
Toba G, Aigaki T. 2000. Disruption of the Microsomal glutathione S-transferase-like gene reduces life span of Drosophila melanogaster. Gene 253: 179-187.

Untergasser A, Cutcutache I, Koressaar T, Ye J, Faircloth BC, Remm M, Rozen SG. 2012. Primer3-new capabilities and interfaces. Nucleic Acids Res 40: e115.

Vibranovski MD, Zhang Y, Long M. 2009. General gene movement off the X chromosome in the Drosophila genus. Genome Res 19: 897-903.

Wang W, Zheng H, Fan C, Li J, Shi J, Cai Z, Zhang G, Liu D, Zhang J, Vang S, et al. 2006. High rate of chimeric gene origination by retroposition in plant genomes. Plant Cell 18: 1791-1802.

Wei W, Gilbert N, Ooi SL, Lawler JF, Ostertag EM, Kazazian HH, Boeke JD, Moran JV. 2001. Human L1 retrotransposition: cis preference versus trans complementation. Mol Cell Biol 21: 1429-1439.

Wicker T, Sabot F, Hua-Van A, Bennetzen JL, Capy P, Chalhoub B, Flavell A, Leroy P, Morgante M, Panaud O, et al. 2007. A unified classification system for eukaryotic transposable elements. Nat Rev Genet 8: 973-982.

Witherspoon DJ, Xing JC, Zhang YH, Watkins WS, Batzer MA, Jorde LB. 2010. Mobile element scanning (ME-Scan) by targeted high-throughput sequencing. BMC Genomics 11: 410.

Yang Z. 2007. PAML 4: phylogenetic analysis by maximum likelihood. Mol Biol Evol 24: 1586-1591.

Yoshioka K, Honma H, Zushi M, Kondo S, Togashi S, Miyake T, Shiba T. 1990. Virus-like particle formation of Drosophila copia through autocatalytic processing. EMBO J 9: 535-541.

Yue F, Cheng Y, Breschi A, Vierstra J, Wu WS, Ryba T, Sandstrom R, Ma ZH, Davis C, Pope BD, et al. 2014. A comparative encyclopedia of DNA elements in the mouse genome. Nature 515: 355-364.

Zhang YE, Landback P, Vibranovski MD, Long M. 2011. Accelerated recruitment of new brain development genes into the human genome. PLoS Biol 9: e1001179.

Zhou Q, Zhang G, Zhang Y, Xu S, Zhao R, Zhan Z, Li X, Ding Y, Yang S, Wang W. 2008. On the origin of new genes in Drosophila. Genome Res 18: $1446-1455$.

Zhu Z, Tan S, Zhang Y, Zhang YE. 2016. LINE-1-like retrotransposons contribute to RNA-based gene duplication in dicots. Sci Rep 6: 24755.

Received January 29, 2016; accepted in revised form October 18, 2016. 


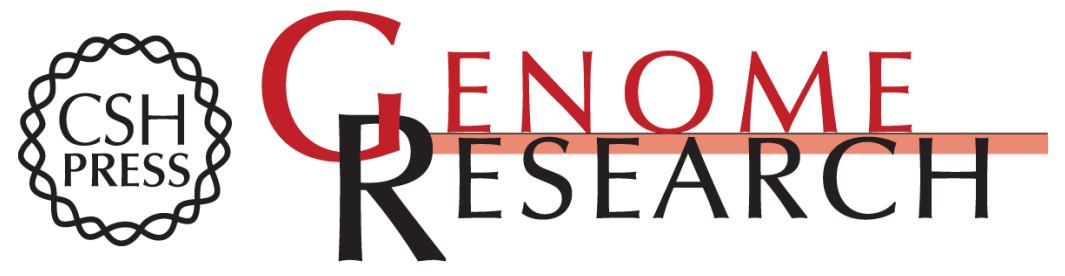

\section{LTR-mediated retroposition as a mechanism of RNA-based duplication in metazoans}

Shengjun Tan, Margarida Cardoso-Moreira, Wenwen Shi, et al.

Genome Res. 2016 26: 1663-1675 originally published online October 20, 2016

Access the most recent version at doi:10.1101/gr.204925.116

Supplemental Material

References

Open Access

Creative Commons

License

Email Alerting Service
http://genome.cshlp.org/content/suppl/2016/11/14/gr.204925.116.DC1

This article cites 87 articles, 31 of which can be accessed free at: http://genome.cshlp.org/content/26/12/1663.full.html\#ref-list-1

Freely available online through the Genome Research Open Access option.

This article, published in Genome Research, is available under a Creative Commons License (Attribution-NonCommercial 4.0 International), as described at http://creativecommons.org/licenses/by-nc/4.0/.

Receive free email alerts when new articles cite this article - sign up in the box at the top right corner of the article or click here.

\section{Affordable, Accurate Sequencing.}

